\title{
Drug-induced hypersensitivity syndrome in a patient with systemic lupus erythematosus and psoriasis
}

\author{
Takako Miura, Toshiyuki Yamamoto
}

Department of Dermatology, Fukushima Medical University, Fukushima, Japan

Corresponding author: Prof. Toshiyuki Yamamoto, E-mail: toyamade@fmu.ac.jp

Sir,

Co-existence of systemic lupus erythematosus (SLE) and psoriasis is relatively rare. We herein describe a rare case of drug-induced hypersensitivity syndrome (DIHS) in a patient with SLE and psoriasis vulgaris, induced by an anticonvulsant.

A 39-year-old man was diagnosed with SLE at the age of 28. Seven years later, he developed psoriasis (Figs. la and $1 \mathrm{~b}$ ), and was treated with topical corticosteroids and vitamin D3 ointment. Moreover, he had aortic regurgitation (AR), and underwent aortic valve replacement. After the operation, he developed convulsions, and carbamazepine (Tegretol $^{\text {R; }} 200 \mathrm{mg} /$ day) was administered. Four weeks later, he developed itchy eruptions on the trunk and extremities, with a fever reaching up to $40^{\circ} \mathrm{C}$. On physical examination, erythema multiforme-like lesions were spread on the trunk and extremities (Fig. lc). Tegretol ${ }^{\mathrm{R}}$ was discontinued, but one week later, erosions of the oral mucosa appeared (Fig. ld), and the erythema on the trunk coalesced with purpura. Inguinal lymphadenopathy was observed. Laboratory data showed a normal white blood cell count $(6300 / \mu \mathrm{l})$, with a differential count of $58 \%$ neutrophils and $21 \%$ eosinophils, elevated AST (66 IU/1) and ALT (116 IU/1) levels. Serum anti-nuclear antibody was positive (1:320, homogenous), whereas anti-DNA antibody was within normal ranges and hypocomplementemia was not detected. A biopsy specimen from the abdomen revealed apoptotic keratinocytes and perivascular inflammatory cell infiltration composed of mononuclear cells and eosinophils in the edematous upper dermis (Fig. 2). Before the onset of drug eruption, the patient's SLE was treated with oral prednisolone $(12.5 \mathrm{mg} /$ day $)$;

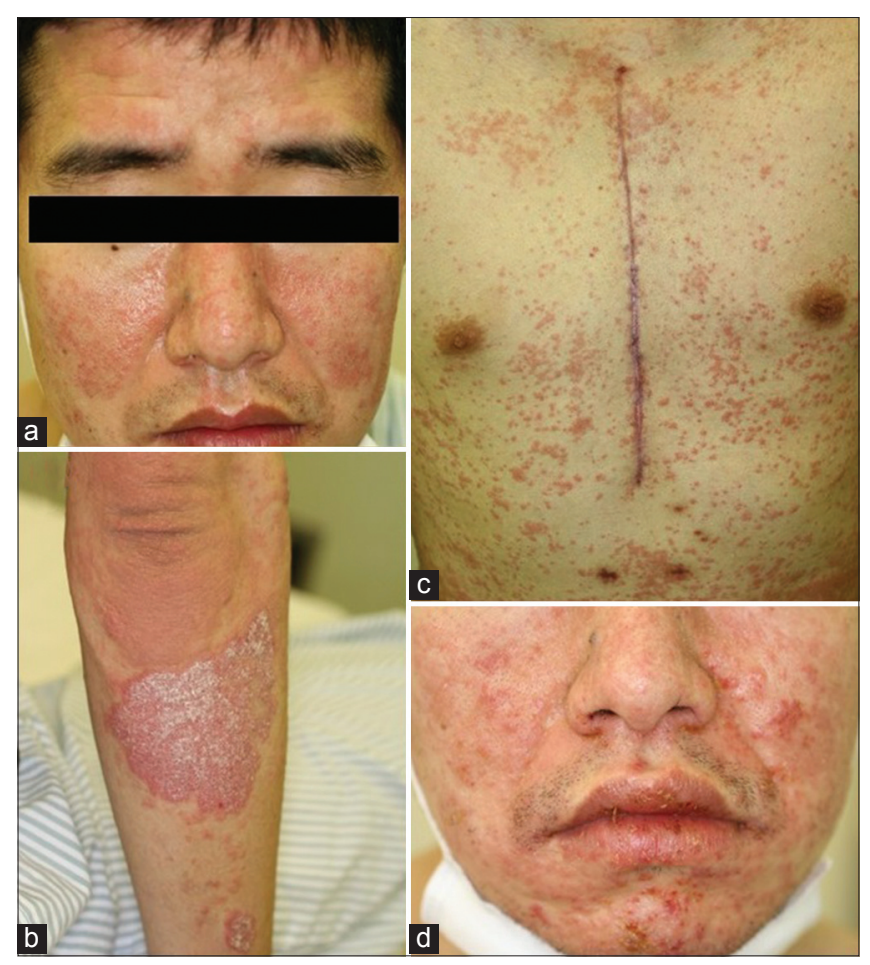

Figure 1: Clinical pictures at initial visit to our department showing malar rash and keratotic plaques on the forearm $(a, b)$. Drug eruption showing erythema multiforme-like erythemas scattered on the trunk (c), facial erythema and mucosal erosion (d).

however the eruption was improved by escalating the dose of prednisolone to $25 \mathrm{mg} /$ day. A lymphocyte transformation test using Tegreto ${ }^{\mathrm{R}}$ was weakly positive (S.I. 188\%), and HHV-6 IgG titer (1:10) was elevated (1:640) six weeks later. HLA-A and -B genotyping revealed HLA-A*1101, A*3101, B*1501, and B*3501.

The co-existence of psoriasis and SLE is sometimes seen, and both diseases share some common pathogenesis, such as Thl/Thl7 type-dominant cytokine imbalance, plasmacytoid dendritic cell activation via Toll-like

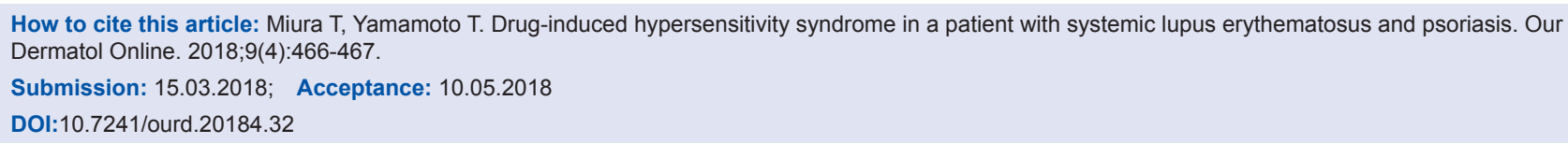




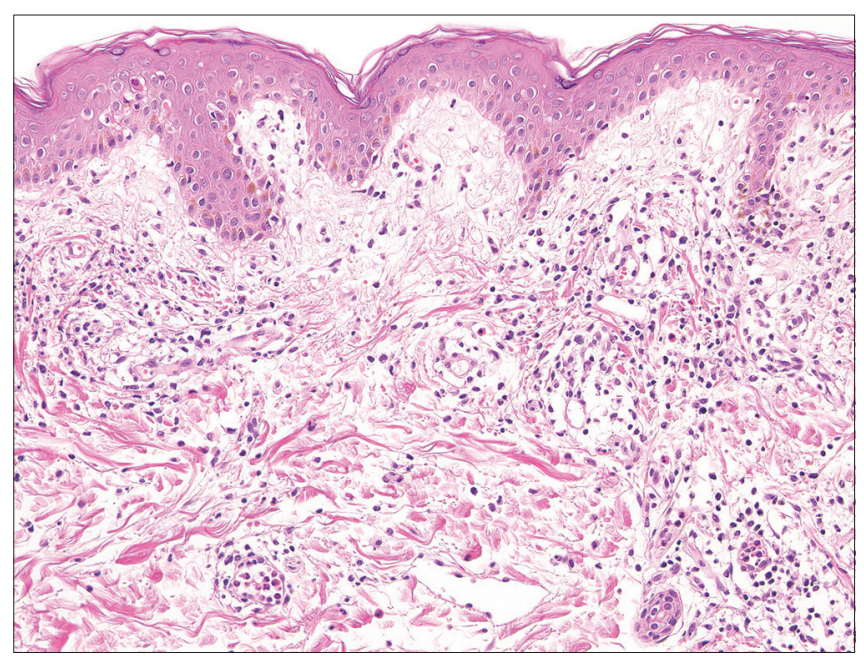

Figure 2: Histological features showing apoptotic cells in the epidermis and cellular infiltrates composed of mononuclear cells and eosinophils in the upper dermis.

receptors, and interferon- $\alpha$ release. Our patient developed psoriasis seven years after the diagnosis of SLE. Furthermore, he had AR and received operation at the remission phase of SLE. Four weeks after administration of an anticonvulsant drug, he developed DIHS, which fulfilled the Japanese criteria, without exacerbation of either SLE or psoriasis. It is known that several autoimmune disorders, including SLE, are induced after DIHS was improved $[1,2]$, possibly due to functional defects of regulatory $\mathrm{T}$ cells (Tregs) [3]. However, our case developed SLE before the occurrence of DIHS, and thus the development of SLE was unassociated with DIHS. Patients with SLE have an increased risk of developing adverse cutaneous drug eruption [4], and cases such as toxic epidermal necrolysis (TEN) and Stevens-Johnson syndrome (SJS) rarely occurs in patients with SLE [5].

To date, only one suspected case of SLE has been reported to develop drug reaction with eosinophilia and systemic symptoms [6]. HLA-A*3101 is significantly associated with susceptibility to carbamazepine-induced adverse drug reactions in Japanese population [7]. Our case also had HLA-A*3101, therefore may have developed DIHS unassociated with SLE itself.

\section{Consent}

The examination of the patient was conducted according to the Declaration of Helsinki principles.

\section{REFERENCES}

1. Kano Y, Tohyama M, Aihara M, Matsukura S, Watanabe H, Sueki $\mathrm{H}$, et al. Sequelae in 145 patients with drug-induced hypersensitivity syndrome/drug reaction with eosinophilia and systemic symptoms: survey conducted by the Asian Reserch Committee on Severe Cutaneous Adverse Reactions (ASCAR). J Dermatol. 2015;42:276-82.

2. Aota N, Hirahara K, Kano Y, Fukuoka T, Yamada A, Shiohara T. Systemic lupus erythematosus presenting with Kikuchi-Fujimoto's disease as a long-term sequela of drug-induced hypersensitivity syndrome: a possible role of Epstein-Barr virus reactivation. Dermatology. 2009;218:275-77.

3. Takahashi R, Kano Y, Yamazaki Y, Kimishima M, Mizukawa Y, Shiohara T. Defective regulatory $\mathrm{T}$ cells in patients with severe drug eruptions: timing of the dysfunction is associated with the pathological phenotype and outcome. J Immunol. 2009;182:8071-9.

4. Hernández-Salazar A, Rosales SP, Rangel-Frausto S, Criollo E, Archer-Dubon C, Orozco-Topete R. Epidemiology of adverse cutaneous drug eruptions: a prospective study in hospitalized patients. Arch Med Res. 2006;37:899-902.

5. Horne NS, Narayan AR, Young RM, Frieri M. Toxic epidermal necrolysis in systemic lupus erythematosus. Autoimmun Rev. 2006;5:160-4.

6. Chen Y-C, Chang C-Y, Cho Y-T, Chiu H-C, Chu C-Y. Long-term sequelae of drug reaction with eosinophilia and systemic symptoms: a retrospective cohort study from Taiwan. J Am Acad Dermatol. 2013;68:459-65.

7. Ozeki T, Mushiroda T, Yowang A, Takahashi A, Kubo M, Shirakata Y, et al. Genome-wide association study identifies HLA-A*3101 allele as a genetic risk factor for carbamazepineinduced cutaneous adverse drug reactions in Japanese population. Hum Mol Genet. 2011;20:1034-41.

Copyright by Takako Miura, et al. This is an open-access article distributed under the terms of the Creative Commons Attribution License, which permits unrestricted use, distribution, and reproduction in any medium, provided the original author and source are credited.

Source of Support: Nil, Conflict of Interest: None declared. 